ELECTRONIC RESEARCH ANNOUNCEMENTS

OF THE AMERICAN MATHEMATICAL SOCIETY

Volume 4, Pages 109-114

S 1079-6762(98)00052-3 (December 15, 1998)

\title{
ON ASPHERICAL PRESENTATIONS OF GROUPS
}

\author{
SERGEI V. IVANOV
}

(Communicated by Efim Zelmanov)

\begin{abstract}
The Whitehead asphericity conjecture claims that if $\langle\mathcal{A} \| \mathcal{R}\rangle$ is an aspherical group presentation, then for every $\mathcal{S} \subset \mathcal{R}$ the subpresentation $\langle\mathcal{A} \| \mathcal{S}\rangle$ is also aspherical. This conjecture is generalized for presentations of groups with periodic elements by introduction of almost aspherical presentations. It is proven that the generalized Whitehead asphericity conjecture (which claims that every subpresentation of an almost aspherical presentation is also almost aspherical) is equivalent to the original Whitehead conjecture and holds for standard presentations of free Burnside groups of large odd exponent, Tarski monsters and some others. Next, it is proven that if the Whitehead conjecture is false, then there is an aspherical presentation $E=\langle\mathcal{A} \| \mathcal{R} \cup z\rangle$ of the trivial group $E$, where the alphabet $\mathcal{A}$ is finite or countably infinite and $z \in \mathcal{A}$, such that its subpresentation $\langle\mathcal{A} \| \mathcal{R}\rangle$ is not aspherical. It is also proven that if the Whitehead conjecture fails for finite presentations (i.e., with finite $\mathcal{A}$ and $\mathcal{R})$, then there is a finite aspherical presentation $\langle\mathcal{A} \| \mathcal{R}\rangle$, $\mathcal{R}=\left\{R_{1}, R_{2}, \ldots, R_{n}\right\}$, such that for every $\mathcal{S} \subseteq \mathcal{R}$ the subpresentation $\langle\mathcal{A} \| \mathcal{S}\rangle$ is aspherical and the subpresentation $\left\langle\mathcal{A} \| R_{1} R_{2}, R_{3}, \ldots, R_{n}\right\rangle$ of aspherical $\left\langle\mathcal{A} \| R_{1} R_{2}, R_{2}, R_{3}, \ldots, R_{n}\right\rangle$ is not aspherical. Now suppose a group presentation $H=\langle\mathcal{A} \| \mathcal{R}\rangle$ is aspherical, $x \notin \mathcal{A}, W(\mathcal{A} \cup x)$ is a word in the alphabet $(\mathcal{A} \cup x)^{ \pm 1}$ with nonzero sum of exponents on $x$, and the group $H$ naturally embeds in $G=\langle\mathcal{A} \cup x \| \mathcal{R} \cup W(\mathcal{A} \cup x)\rangle$. It is conjectured that the presentation $G=\langle\mathcal{A} \cup x \| \mathcal{R} \cup W(\mathcal{A} \cup x)\rangle$ is aspherical if and only if $G$ is torsion free. It is proven that if this conjecture is false and $G=\langle\mathcal{A} \cup x \| \mathcal{R} \cup W(\mathcal{A} \cup x)\rangle$ is a counterexample, then the integral group ring $\mathbb{Z}(G)$ of the torsion free group $G$ will contain zero divisors. Some special cases where this conjecture holds are also indicated.
\end{abstract}

Let

$$
\langle\mathcal{A} \| \mathcal{R}\rangle
$$

be a group presentation, where $\mathcal{A}$ is an alphabet, and $\mathcal{R}$ is a set of defining relators (which are words in $\mathcal{A}^{ \pm 1}=\mathcal{A} \cup \mathcal{A}^{-1}$ ). The group $G$ given by this presentation is the quotient $F(\mathcal{A}) / N(\mathcal{R})$, where $F(\mathcal{A})$ is the free group over the alphabet $\mathcal{A}$ and $N(\mathcal{R})$ is the normal closure of $\mathcal{R}$ in $F(\mathcal{A})$. The quotient $N(\mathcal{R}) / N^{\prime}(\mathcal{R})$, where $N^{\prime}(\mathcal{R})$ is the commutator subgroup of $N(\mathcal{R})$, can be turned into a (left) $G$-module as follows: If $\alpha: F(\mathcal{A}) \rightarrow G$ and $\beta: N(\mathcal{R}) \rightarrow N(\mathcal{R}) / N^{\prime}(\mathcal{R})$ are natural epimorphisms, and $W \in F(\mathcal{A}), S \in N(\mathcal{R})$, then

$$
W^{\alpha} \cdot S^{\beta}=\left(W S W^{-1}\right)^{\beta} .
$$

Received by the editors April 13, 1998.

1991 Mathematics Subject Classification. Primary 20F05, 20F06, 20F32; Secondary 57M20.

Supported in part by an Alfred P. Sloan Research Fellowship and NSF grant DMS 95-01056.

(c)1998 American Mathematical Society 
Clearly, this $G$-action extends to an action of the integral group ring $\mathbb{Z}(G)$ of $G$ over $\mathcal{M}(G)=N(\mathcal{R}) / N^{\prime}(\mathcal{R})$ by setting

$$
\left(W_{1}^{\alpha} \pm W_{2}^{\alpha}\right) \cdot S^{\beta}=\left(W_{1} S W_{1}^{-1} W_{2} S^{ \pm 1} W_{2}^{-1}\right)^{\beta} .
$$

This $\mathbb{Z}(G)$-module $\mathcal{M}(G)$ is called the relation module of $G=\langle\mathcal{A} \| \mathcal{R}\rangle$. A group presentation (1) is called aspherical if its relation module is freely generated by the images $R^{\beta}$ of relators $R \in \mathcal{R}$. If $K_{G}$ is a 2-complex associated with $G=\langle\mathcal{A} \| \mathcal{R}\rangle$ in a standard way $\left(K_{G}\right.$ has a single 0 -cell and $\pi_{1}\left(K_{G}\right)=G$ ), then $G$ is aspherical if and only if so is $K_{G}$ (see [GR]; we recall that a 2-complex $K$ is called aspherical if $\left.\pi_{2}(K)=0\right)$.

The Whitehead asphericity conjecture (originally stated as a question in [W]) claims that every subcomplex of an aspherical 2-complex $K$ is also aspherical. The problem has received a great deal of attention (see [GR], [H1], [H2], [Hb2], [Lf] and references there) but still is far from being solved. A remarkable result on this conjecture proven by Howie [H1] reduces the conjecture to asphericity of subcomplexes of aspherical contractible 2-complexes. More specifically, it was shown in [H1] that if the Whitehead conjecture is false, then there is a counterexample of one of the following two types:

1. $K$ is a finite aspherical contractible 2 -complex and $L$ is nonaspherical subcomplex of $K$ obtained from $K$ by removing one 2-cell.

2. $K$ is an aspherical contractible 2-complex, $K=\bigcup_{i=1}^{\infty} L_{i}, \quad L_{i} \subset L_{i+1}$, the inclusion $L_{i} \rightarrow L_{i+1}$ is nullhomotopic, and each $L_{i}$ is finite and is not aspherical.

Recently Luft [Lf] reproved Howie's result and showed that the existence of a counterexample of type 1 implies the existence of a counterexample of type 2 .

Clearly, in group-theoretic terms the Whitehead conjecture is rephrased as follows: If presentation (1) is aspherical, then every subpresentation of (1) of the form $\left\langle\mathcal{A} \| \mathcal{R}^{\prime}\right\rangle$ with $\mathcal{R}^{\prime} \subset \mathcal{R}$ is also aspherical.

First we will see that it is possible to assume in the Whitehead asphericity conjecture that the removed part $\mathcal{R} \backslash \mathcal{R}^{\prime}$ of $\mathcal{R}$ is just a letter of $\mathcal{A}$. We recall that a presentation is called finite if both $\mathcal{A}$ and $\mathcal{R}$ are finite.

Theorem 1. If the Whitehead asphericity conjecture is false, then there is an aspherical presentation $E=\langle\mathcal{A} \| \mathcal{R} \cup z\rangle$ of the trivial group $E$, where the alphabet $\mathcal{A}$ is finite or countably infinite and $z \in \mathcal{A}$, such that its subpresentation $\langle\mathcal{A} \| \mathcal{R}\rangle$ is not aspherical. In addition, if there is a finite presentation giving a counterexample to the Whitehead asphericity conjecture, then there is a finite presentation $\langle\mathcal{A} \| \mathcal{R} \cup z\rangle$ such that its subpresentation $\langle\mathcal{A} \| \mathcal{R}\rangle$ is not aspherical.

We recall that elementary Andrews-Curtis transformations over a finite group presentation $\langle\mathcal{A} \| \mathcal{R}\rangle$ of types (T1)-(T3) are defined as follows:

(T1) Add a new letter $b \notin \mathcal{A}$ to both $\mathcal{A}$ and $\mathcal{R}$.

(T2) If $a \in \mathcal{A}, a \in \mathcal{R}$, and $a, a^{-1}$ do not occur in relators $R \in \mathcal{R} \backslash a$, then delete $a$ in both $\mathcal{A}$ and $\mathcal{R}$.

(T3) Replace $R \in \mathcal{R}$ by $C_{1} R^{\varepsilon} C_{1}^{-1} C_{2} S^{\delta} C_{2}^{-1}$, where $\varepsilon, \delta= \pm 1, C_{1}, C_{2} \in F(\mathcal{A})$, and $S \in \mathcal{R} \backslash R$.

Two finite presentations are called Andrews-Curtis equivalent if one of them can be obtained from the other by a finite sequence of elementary Andrews-Curtis 
transformations. (We recall that another major problem of low-dimensional topology, the so-called Andrews-Curtis conjecture [AC], asks whether a finite aspherical presentation of the trivial group is Andrews-Curtis equivalent to $\langle\mathcal{A} \| \mathcal{A}\rangle$.)

Clearly, transformations (T1)-(T3) preserve the asphericity of a presentation $\langle\mathcal{A} \| \mathcal{R}\rangle$. Moreover, (T1)-(T2) evidently preserve the asphericity of subpresentations. Whether (T3) preserves the asphericity of subpresentations is unclear and turns out to be equivalent to the Whitehead asphericity conjecture for finite presentations following from

Theorem 2. Suppose $\langle\mathcal{A} \| \mathcal{R}\rangle$ is a finite aspherical presentation. Then $\langle\mathcal{A} \| \mathcal{R}\rangle$ is Andrews-Curtis equivalent (with a single (T1), no (T2) and several (T3)'s) to a finite aspherical presentation $\langle\mathcal{B} \| \mathcal{S}\rangle$ such that for every $\mathcal{S}^{\prime} \subseteq \mathcal{S}$ the subpresentation $\left\langle\mathcal{B} \| \mathcal{S}^{\prime}\right\rangle$ is aspherical.

For 2-complexes (for definitions see [S] or [H2]) Theorem 2 implies

Corollary. Every finite aspherical 2-complex can be 3-deformed to a finite 2-complex all of whose subcomplexes are aspherical.

Technical details in proving Theorem 2 enable us to sharpen the equivalence between the Whitehead asphericity conjecture for finite presentations and preservation of asphericity of subpresentations under (T3) as follows:

Theorem 3. If the Whitehead asphericity conjecture is false for finite presentations, then there is a finite aspherical presentation $\langle\mathcal{A} \| \mathcal{R}\rangle, \mathcal{R}=\left\{R_{1}, R_{2}, \ldots, R_{n}\right\}$, such that for every $\mathcal{S} \subseteq \mathcal{R}$ the subpresentation $\langle\mathcal{A} \| \mathcal{S}\rangle$ is aspherical and the subpresentation $\left\langle\mathcal{A} \| R_{1} R_{2}, R_{3}, \ldots, R_{n}\right\rangle$ of aspherical $\left\langle\mathcal{A} \| R_{1} R_{2}, R_{2}, R_{3}, \ldots, R_{n}\right\rangle$ is not aspherical.

It is well known and easy to show that if (1) is aspherical, then none of $R \in \mathcal{R}$ is a proper power in the free group $F(\mathcal{A})$ over $\mathcal{A}$ and the group $G$ is torsion free. In particular, presentations of periodic groups (like those of free Burnside groups, Tarski monsters and others constructed in [O1]-[O3], [IO1], [I], [IO2]) seem to have nothing to do with aspherical presentations and the Whitehead conjecture. This, however, is not the case and we will see that the Whitehead conjecture has a natural expansion to groups with periodic elements. More specifically, let a group $G$ be given by presentation (1). For every $R \in \mathcal{R}$ we let $R=Q_{R}^{m_{R}}$ in the free group $F(\mathcal{A})$, where $Q_{R}$ is not proper in $F(\mathcal{A})$ and $m_{R} \geq 1$. Following [GR], we call presentation (1) almost aspherical if defining relations of $\mathcal{M}(G)$ (with generating set $\left.\left\{R^{\beta} \mid R \in \mathcal{R}\right\}\right)$ look like

$$
\left(1-Q_{R}^{\alpha}\right) \cdot R^{\beta}=0, \quad R \in \mathcal{R}
$$

(note that if none of $R$ is a proper power, that is, $m_{R}$ are all 1 , then we have the foregoing definition of an aspherical presentation for $1-Q_{R}^{\alpha}=1-R^{\alpha}=0$ in the integral group ring $\mathbb{Z}(G)$ of $G)$.

For example, using this definition, one can restate the main result of Lyndon's article [Ln] as follows: A one-relator group presentation is almost aspherical. Another interesting example of an almost aspherical presentation is constructed by induction on $i \geq 0$ as follows: Let $\mathcal{A}^{ \pm 1}=\left\{a_{1}^{ \pm 1}, \ldots, a_{m}^{ \pm 1}\right\}$ and $F_{m}=F(\mathcal{A})$ be the free group of rank $m$ over $\mathcal{A}$. Following Ol'shanskii [O1] (and [I]), put $B(m, n, 0)=F_{m}$. Assuming that $B(m, n, i), i \geq 0$, is already constructed as a quotient group of $F_{m}$, define $A_{i+1}$ to be a shortest word over $\mathcal{A}^{ \pm 1}$ (if any) whose image in $B(m, n, i)$ has 
infinite order. Then $B(m, n, i+1)$ is the quotient group of $B(m, n, i)$ by the relation $A_{i+1}^{n}=1$. Clearly,

$$
B(m, n, i+1)=\left\langle\mathcal{A} \| A_{1}^{n}, A_{2}^{n}, \ldots, A_{i+1}^{n}\right\rangle
$$

provided the word $A_{i+1}$ exists (otherwise, $B(m, n, i)$ is periodic and the construction stalls). It is shown in [O1] that if $m>1, n$ is odd and $n>10^{10}$, then $A_{i+1}$ always exists and the group

$$
B(m, n, \infty)=\left\langle\mathcal{A} \| A_{1}^{n}, A_{2}^{n}, \ldots, A_{i+1}^{n}, \ldots\right\rangle
$$

obtained by imposing on $F_{m}$ of all defining relations $A_{i}^{n}=1$ for $i=1,2, \ldots$ is naturally isomorphic to the free Burnside group $B(m, n)$ with $m$ generators and exponent $n$ (recall that $B(m, n)$ is the quotient $F_{m} / F_{m}^{n}$, where $F_{m}^{n}$ is the subgroup of $F_{m}$ generated by all $n$th powers; the same results are proven in [I] for $m>1$ and all $n \geq 2^{48}$ such that if $n$ is even, then $n$ is divisible by $\left.2^{9}\right)$.

Moreover, it is shown by Ashmanov and Ol'shanskii [AO] (see also Chapter 10 in [O3]) that (2) is an almost aspherical presentation provided $m>1, n>10^{10}$ is odd (for even $n$ this is not the case). It is worth mentioning that Ol'shanskii's presentations of Tarski monsters [O2] (see also [O3]; we recall that a Tarski monster is an infinite group all of whose proper subgroups are cyclic of the same prime order $p$ ) are also almost aspherical. Hence, in analogy with the Whitehead conjecture, one might wonder if all subpresentations of (2) (or of Tarski monster's given in [O2]) are also almost aspherical. More generally, one could generalize the Whitehead asphericity conjecture as follows.

Conjecture 1. Every subpresentation of an almost aspherical group presentation is also almost aspherical.

Surprisingly, this turns out not to be any more general.

Theorem 4. This generalized Whitehead asphericity conjecture is equivalent to the original Whitehead asphericity conjecture.

As a matter of fact, when proving Theorem 4, we make reduction to an aspherical presentation of the trivial group (similar to reductions in [H1], [Lf]) which yields the following.

Theorem 5. If the generalized Whitehead asphericity conjecture is false, then there exists a counterexample $\langle\mathcal{A} \| \mathcal{R}\rangle$ to the Whitehead asphericity conjecture such that $\langle\mathcal{A} \| \mathcal{R}\rangle$ is an aspherical presentation of the trivial group, where $\mathcal{A}$ is finite or countably infinite and $\mathcal{R}$ contains a finite subset $\mathcal{R}^{\prime}$ so that the subpresentation $\left\langle\mathcal{A} \| \mathcal{R}^{\prime}\right\rangle$ is not aspherical.

In view of Theorems $4-5$, the problem whether the generalized Whitehead asphericity conjecture holds for presentations (2) (and other group presentations of [O3], [IO1]) becomes especially interesting. A positive solution to this problem is provided by

Theorem 6. (a) Let $m>1, n$ odd, $n>10^{10}$. Then every subpresentation of the free Burnside group $B(m, n)$ presented by (2) is almost aspherical.

(b) Let $G=\langle\mathcal{A} \| \mathcal{R}\rangle$ be a (graded) presentation constructed as in Chapters 6 and 8 of [O3]. Then every subpresentation of $\langle\mathcal{A} \| \mathcal{R}\rangle$ is almost aspherical. In particular, if $p$ is prime, $p \gg 1$, then every subpresentation of Tarski monster presentation constructed in $[\mathrm{O} 2]$ is almost aspherical. 
Now consider a special type of group presentations:

$$
G=\langle\mathcal{A} \cup x \| \mathcal{R}(\mathcal{A}) \cup W(\mathcal{A} \cup x)\rangle
$$

where $x \notin \mathcal{A}$, all relators in $\mathcal{R}(\mathcal{A})$ are words in $\mathcal{A}^{ \pm 1}$ and $W(\mathcal{A} \cup x)$ is a word in $(\mathcal{A} \cup x)^{ \pm 1}$ with nonzero sum of exponents on $x$. Assuming that (3) is aspherical, one can easily reduce the problem on asphericity of subpresentation $\langle\mathcal{A} \| \mathcal{R}\rangle$ to whether or not the group $H=\langle\mathcal{A} \| \mathcal{R}\rangle$ embeds in the group $G$ given by (3), that is, whether the equation $W(\mathcal{A} \cup x)=1$ is solvable over $H$. This, however, is another difficult open problem (the so-called Kervaire problem about the solvability of equations over groups) and the affirmative solution is known only in some special cases. For example: If all occurrences of $x^{ \pm 1}$ in $W$ have positive (or negative) exponents (Levin [Lv]); If the sum of exponents on $x$ in $W$ is \pm 1 and $H$ is torsion free (Klyachko $[\mathrm{K}])$; If $H$ is locally indicable, that is, every nontrivial finitely generated subgroup of $H$ has an infinite cyclic epimorphic image (Howie [H3]; see also Brodskii [B]).

The following seems worth mentioning and is immediate from the foregoing reduction and Klyachko's result.

Proposition. If (3) is a balanced presentation of the trivial group (and hence aspherical), then its subpresentation $H=\langle\mathcal{A} \| \mathcal{R}(A)\rangle$ is aspherical if and only if $H$ is a torsion free group.

Now let us turn tables around to indicate an interesting connection between the asphericity of presentation (3), torsion in the group $G$, and the Kaplansky problem on zero divisors which asks whether the group ring of a torsion free group over an integral domain can have zero divisors.

First let us state a conjecture that, like the Whitehead asphericity conjecture, is actually a problem more convenient to state in the affirmative form.

Conjecture 2. Suppose a group presentation $H=\langle\mathcal{A} \| \mathcal{R}\rangle$ is aspherical, $x \notin \mathcal{A}$, $W(\mathcal{A} \cup x)$ is a word in $(\mathcal{A} \cup x)^{ \pm 1}$ with nonzero sum of exponents on $x$, and the group $H$ naturally embeds in

$$
G=\langle\mathcal{A} \cup x \| \mathcal{R} \cup W(\mathcal{A} \cup x)\rangle .
$$

Then presentation (4) is aspherical if and only if the group $G$ is torsion free.

Theorem 7. If Conjecture 2 fails and $G=\langle\mathcal{A} \cup x \| \mathcal{R} \cup W(\mathcal{A} \cup x)\rangle$ is a counterexample to it, then the group $G$ is a torsion free group whose integral group ring $\mathbb{Z}(G)$ contains zero divisors. In addition, if $W(\mathcal{A} \cup x)$ has $n$ occurrences of $x^{ \pm 1}$, then $\mathbb{Z}(G)$ contains a zero divisor $Z$ with $|\operatorname{supp} Z| \leq n$.

Some special cases where Conjecture 2 holds are indicated in

Theorem 8. Conjecture 2 holds in the following cases:

(a) The group $H=\langle\mathcal{A} \| \mathcal{R}\rangle$ is locally indicable.

(b) The sum of exponents on $x$ in $W(\mathcal{A} \cup x)$ is \pm 1 .

(c) The number of occurrences of $x^{ \pm 1}$ in $W(\mathcal{A} \cup x)$ is at most 3 .

(d) If $W(\mathcal{A} \cup x) \equiv U_{1} x^{\varepsilon_{1}} \ldots U_{n} x^{\varepsilon_{n}}$, where $\varepsilon_{1}, \ldots, \varepsilon_{n} \in\{ \pm 1\}, U_{1}, \ldots, U_{n}$ are words in $\mathcal{A}^{ \pm 1}$, then there are precisely two alternations of sign in the cyclic sequence $\left(\varepsilon_{1}, \ldots, \varepsilon_{n}\right)$ and if $\varepsilon_{k} \varepsilon_{k+1}=\varepsilon_{\ell} \varepsilon_{\ell+1}=-1$ (subscripts $\left.(\bmod n)\right)$ with $k \neq \ell$, then $U_{k+1} \neq 1, U_{\ell+1} \neq 1$ in $H=\langle\mathcal{A} \| \mathcal{R}\rangle$. 
Proofs of Theorems 1-8 make use of (more or less) standard techniques of group theory such as Nielsen reduced bases for subgroups of free groups, ReidemeisterSchreier rewriting process, Fox's derivatives, small cancellation theory, Ol'shanskii's machinery of graded diagrams and graded group presentations, and van Kampen diagrams on orientable surfaces.

\section{REFERENCES}

[AO] I.S. Ashmanov and A.Yu. Ol'shanskii, On abelian and central extensions of aspherical groups, Izv. Vyssh. Uchebn. Zaved. Mat. 11 (1985), 48-60. MR 87m:20095

[AC] J.J. Andrews and M.L. Curtis, Free groups and handlebodies, Proc. Amer. Math. Soc. 16 (1965), 192-195. MR 30:3454

[B] S.D. Brodskii, Equations over groups and groups with a single defining relation, Uspekhi Mat. Nauk 35 (1980), 183. MR 82a:20041

[GR] M. Gutierrez and J.G. Ratcliffe, On the second homotopy group, Quart. J. Math. Oxford 32 (1981), 45-55. MR 82g:57003

[H1] J. Howie, Some remarks on a problem of J.H.C. Whitehead, Topology 22 (1983), 475-485. MR 85g:57003

[H2] J. Howie, On the asphericity of ribbon disc complements, Trans. Amer. Math. Soc. 289 (1985), 281-302. 87a:57007

[H3] J. Howie, On locally indicable groups, Math. Z. 180 (1982), 445-461. MR 84b:20036

[Hb1] J. Huebschmann, Cohomology theory of aspherical groups and of small cancellation groups, J. Pure Appl. Algebra 14 (1979), 137-143. MR 80e:20064

[Hb2] J. Huebschmann, Aspherical 2-complexes and an unsettled problem of J.H.C. Whitehead, Math. Ann. 258 (1981), 17-37. MR 83e:57004

[I] S.V. Ivanov, The free Burnside groups of sufficiently large exponents, Internat. J. Algebra Comp. 4 (1994), 1-308. MR 95h:20051

[IO1] S.V. Ivanov and A.Yu. Ol'shanskii, Some applications of graded diagrams in combinatorial group theory, London Math. Soc. Lecture Note Ser. 160 (1991), 258-308. MR 92j:20022

[IO2] S.V. Ivanov and A.Yu. Ol'shanskii, Hyperbolic groups and their quotients of bounded exponents, Trans. of the Amer. Math. Soc 348 (1996), 2091-2138. MR 96m:20057

[K] A. Klyachko, A funny property of sphere and equations over groups, Comm. Algebra 122 (1994), 1475-1488. MR 94c:20070

[Lv] F. Levin, Solutions of equations over groups, Bull. Amer. Math. Soc. 62 (1962), 603-604. MR 26:212

[Lf] E. Luft, On 2-dimensional aspherical complexes and a problem of J.H.C. Whitehead, Math. Proc. Cambridge Phil. Soc. 119 (1996), 493-495. MR 96h:57003

[Ln] R.C. Lyndon, Cohomology theory of groups with a single defining relation, Ann. Math. 52 (1950), 650-655. MR 13:819b

[LS] R.C. Lyndon and P.E. Schupp, Combinatorial group theory, Springer-Verlag, 1977. MR $\mathbf{5 8 : 2 8 1 8 2}$

[O1] A.Yu. Ol'shanskii, On the Novikov-Adian theorem, Mat. Sbornik 118 (1982), 203-235. MR 83m:20058

[O2] A.Yu. Ol'shanskii, Groups of bounded period with subgroups of prime order, Algebra i Logika 21 (1982), 553-618. MR 85g:20052

[O3] A.Yu. Ol'shanskii, Geometry of defining relations in groups, English translation in Math. and Its Applications (Soviet series), 70, Kluwer Acad. Publishers, 1991 (1989). MR 93g:20071

[S] A.J. Sieradski, Combinatorial isomorphisms and combinatorial homotopy equivalences, J. Pure Appl. Algebra 7 (1976), 59-65. MR 53:9227

[W] J.H.C. Whitehead, On adding relations to homotopy groups, Ann. Math. 42 (1941), 409428. MR 2:323c

Department of Mathematics, University of Illinois at Urbana-Champaign, 1409 West Green Street, Urbana, IL 61801

E-mail address: ivanov@math.uiuc.edu 